\title{
Growth of 2D MoP single crystals on liquid metals by chemical vapor deposition
}

\author{
Feifei Cao ${ }^{1}$, Shuting Zheng ${ }^{2}$, Jingjing Liang ${ }^{1}, \mathrm{Zhi} \mathrm{Li}^{3}$, Bin Wei ${ }^{4}$, Yiran Ding ${ }^{1}$, Zhongchang Wang ${ }^{4}$, \\ Mengqi Zeng ${ }^{2^{*}}, \mathrm{Nan} \mathrm{Xu}^{1^{*}}$ and Lei $\mathrm{Fu}^{1,2^{*}}$
}

\begin{abstract}
Two-dimensional (2D) transition metal phosphides (TMPs) are predicted with many novel properties and various applications. As a member of TMPs family, molybdenum phosphide (MoP) exhibits many exotic physicochemical properties. However, the synthesis of high-quality 2D MoP single crystals is not reported due to the lack of reliable fabrication method, which limits the exploration of $2 \mathrm{D}$ MoP. Here, we report the growth of high-quality ultrathin MoP single crystals with thickness down to $10 \mathrm{~nm}$ on liquid metals via chemical vapor deposition (CVD). The smooth surface of liquid $\mathrm{Ga}$ is regarded as a suitable growth substrate for producing $2 \mathrm{D} \mathrm{MoP}$ single crystals. The Mo source diffuses toward the Ga surface due to the high surface energy to react with phosphorus source, thus to fabricate ultrathin MoP single crystals. Then, we study the second harmonic generation (SHG) of 2D MoP for the first time due to its intrinsic noncentrosymmetric structure. Our study provides an new approach to synthesize and explore other 2D TMPs for future applications.
\end{abstract}

Keywords: 2D materials, transition metal phosphides, molybdenum phosphides, liquid metals, chemical vapor deposition

\section{INTRODUCTION}

Researches in two-dimensional (2D) materials have increasingly grown triggered by pioneering studies on graphene [1-4]. As an emerging family of 2D materials, 2D transition metal phosphides (TMPs) are predicted with novel properties, which are appealing for many applications, including optical, thermal insulating materials, magnetic material and catalysts [5,6]. Thus the reliable synthesis of 2D TMPs is highly demanded.
As a member of TMPs family, molybdenum phosphide $(\mathrm{MoP})$ exhibits many exotic properties with the presence of novel three-component fermions, such as intrinsic non-centrosymmetric structure, high conductivity, strong anisotropic lattice thermal conductivity, superconductivity, and the transformation to a direct bandgap semiconductor in monolayer MoP [7-11]. Yet, traditional solution approaches usually obtain polycrystalline nanoparticles $[12,13]$. The preparation of high-quality 2D MoP single crystals has not yet been reported due to the lack of reliable fabrication methods. Chemical vapor deposition (CVD) approach is considered to be one of the most promising methods to obtain high-quality $2 \mathrm{D}$ single crystals [14]. The emerging liquid metals possess atomically smooth surface, which are regarded as suitable growth substrates for producing 2D materials [15].

In this study, we firstly report the successful growth of high-quality $2 \mathrm{D}$ MoP single crystals via CVD by using liquid gallium $(\mathrm{Ga})$ surface as the growth substrate. Our group has successfully prepared a series of $2 \mathrm{D}$ materials on liquid metal surface [16-19]. Mo source supplied by Mo foil can diffuse toward the Ga surface due to the high surface energy. This enables a well-designed chemical reaction with phosphorus source on the Ga surface, thus to fabricate ultrathin MoP single crystals. Due to the intrinsic non-centrosymmetric structure, MoP would exhibit second harmonic generation (SHG), which is an essential building block for future laser optics, optical communications, and photonic circuits [20]. Therefore, we explore the SHG property of the as-prepared highquality $2 \mathrm{D}$ MoP single crystals for the first time, which reveals their potential as ultrathin frequency doubling

\footnotetext{
${ }^{1}$ Institute for Advanced Studies (IAS), Wuhan University, Wuhan 430072, China

${ }^{2}$ College of Chemistry and Molecular Sciences, Wuhan University, Wuhan 430072, China

${ }^{3}$ MIIT Key Laboratory of Advanced Display Materials and Devices, Ministry of Industry and Information Technology, Institute of Optoelectronics \& Nanomaterials, Nanjing University of Science and Technology, Nanjing 210094, China

${ }^{4}$ Department of Quantum and Energy Materials, International Iberian Nanotechnology Laboratory (INL), 4715-330 Braga, Portugal

* Corresponding authors (emails: zengmq_lan@whu.edu.cn (Zeng M); nxu@whu.edu.cn (Xu N); leifu@whu.edu.cn (Fu L))
} 
crystals in nonlinear optical (NLO) fields. We hope this study of growing high-quality 2D MoP single crystals on liquid metals would provide an approach to synthesize and explore many other 2D TMPs for future applications.

\section{EXPERIMENTAL SECTION}

\section{CVD growth of 2D MoP single crystals on liquid Ga}

A commercial gallium (Ga) pellet (Alfa Aesar, $>99.9 \%$ purity) was departed into small Ga droplets in hot ethanol. Molybdenum (Mo) foil (Alfa Aesar, 99.95 wt.\% purity) substrate was rinsed sequentially in acetone, ethanol and ultrapure water. A Ga droplet was put on the Mo foil $(1 \mathrm{~cm} \times 1 \mathrm{~cm})$ to get $\mathrm{Ga}-$ Mo substrate, which was then heated in a quartz tube to $1100^{\circ} \mathrm{C}$ with a rate of $30^{\circ} \mathrm{C} \mathrm{min}^{-1}$. After that, red phosphorus powder (Alfa Aesar, $>99.9 \%$ purity) was introduced to the tube furnace and the temperature was maintained at $500^{\circ} \mathrm{C}$. The growth process was remained for $30 \mathrm{~min}$ under the flow of $\mathrm{Ar}(200 \mathrm{sccm})$ and $\mathrm{H}_{2}(100 \mathrm{sccm})$. At last, the substrates with grown MoP samples were rapidly cooled to room temperature.

\section{Transferring MoP to the target substrates}

The substrate with the as-grown MoP was firstly spincoated with a polymethyl methacrylate (PMMA) film. Then, PMMA/MoP film was released via soaking Ga in the hydrochloric $(\mathrm{HCl})$ solution (the volume ratio of $\mathrm{HCl}$ : $\mathrm{H}_{2} \mathrm{O}$ is $1: 4$ ) for $4 \mathrm{~h}$. After that, the film was repeatedly washed in the ultrapure water to get rid of the etchant and residues. Afterward, PMMA/MoP film can be transferred to various target substrates, such as $300 \mathrm{~nm}$ $\mathrm{SiO}_{2} / \mathrm{Si}$ substrates and copper $(\mathrm{Cu})$ TEM grid. Finally, PMMA layer was removed by hot acetone.

\section{Raman and SHG measurements}

Raman spectrum was acquired using a WITec Alpha300 R Raman system at an excitation wavelength of $532 \mathrm{~nm}$. The Raman peak of $\mathrm{Si}$ at $520 \mathrm{~cm}^{-1}$ was served as the reference for wavenumber calibration in this characterization. SHG measurements were based on the WITec Alpha300 R confocal Raman system using an excitation source of chameleon Ti: Sapphire femtosecond tunable laser $(\approx 140 \mathrm{fs}, 80 \mathrm{MHz})$. The pulse laser was focused on the sample via objective lens and the SHG signals were then collected in reflection mode by the same objective, which was then passed through a beam splitter and shortpass edge filter $(l=750 \mathrm{~nm})$. The fundamental laser was filtered out and the SHG signal was eventually detected by the spectrometer and charge coupled device (CCD) camera. The pulse laser was linearly polarized along horizontal direction. In order to avoid the influence of dichroic beam splitter on laser polarization ratio and polarized SHG signals, the sample was rotated with a step of $10^{\circ}$ for the angle-resolved SHG measurement. With the help of a tunable analyzer on the collection beam path, the polarized SHG signal was then collected with either parallel or perpendicular configurations and then plotted out with the function of excitation angles.

\section{Density functional theory (DFT) calculations}

The second-order NLO susceptibility of MoP was calculated to reveal the optical property of MoP. First-principles calculation with sum-over-state (SOS) approximation was performed to calculate the frequencydependent SHG susceptibility. For the electronic structure, the first-principles calculation based on DFT was conducted within a primitive cell with a $40 \times 40 \times 40 \mathrm{k}$ point grid and $500 \mathrm{eV}$ energy cutoff. Projector augmented wave pseudopotentials with Perdew, Burke, and Ernzerhof (PBE) exchange correlation were utilized in this process.

\section{Characterizations}

The obtained samples were examined using scanning electron microscopy (SEM) (ZEISS Merlin Compact SEM), X-ray photoelectron spectroscopy (XPS) (Thermo Scientific, ESCALAB 250Xi, monochromatic Al Ka radiation), atomic force microscopy (AFM) (NT-MDT Ntegra Spectra). Transmission electron microscopy (TEM) images were recorded on an aberration-corrected high-resolution TEM system (FEI Titan Themis 200, operating voltage of $80 \mathrm{kV}$ ) and a probe-corrected highresolution TEM (HRTEM) system (Titan Probe corrected TEM, Titan G2 60-300, operating voltage of $300 \mathrm{kV}$ ). The scanning TEM (STEM) were recorded by probe Cs-corrected TEM (FEI Titan ChemiSTEM, operating voltage of $200 \mathrm{kV}$ ) with the high-angle annular dark-field (HAADF) and the bright-field (BF) images. Meanwhile, the elemental mapping of samples was collected by energy dispersive X-ray spectroscopic (EDX).

\section{RESULTS AND DISCUSSION}

High-quality MoP single crystals were synthesized by a CVD method. A piece of Mo foil was used to support the Ga substrate and provide Mo source, while red phosphorus served as phosphorus source (Fig. 1a). The high growth temperature allows the Mo source to diffuse toward the liquid metal Ga surface due to the high surface energy [15]. Then, Mo reacts with phosphorus on the Ga 
a

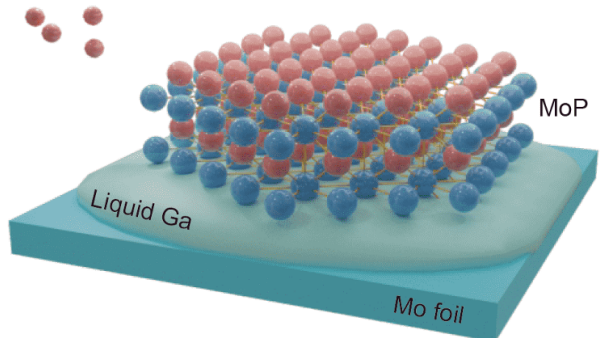

d

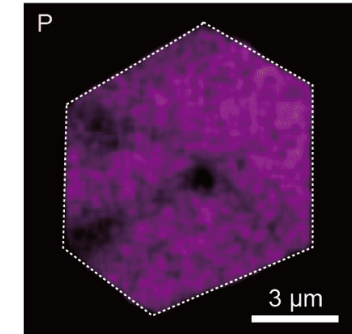

b

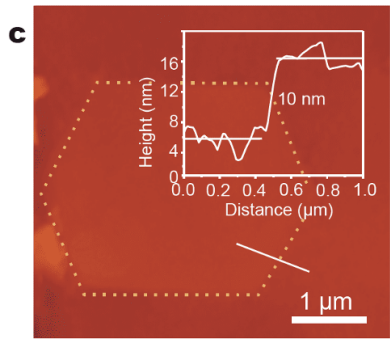

$1 \mu \mathrm{m}$

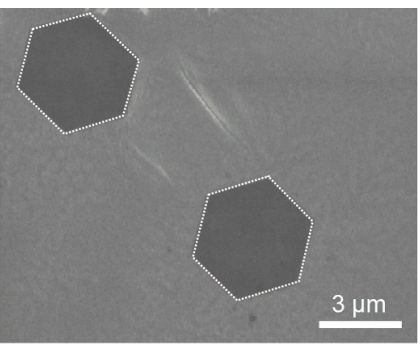

Figure 1 Morphology and chemical composition of 2D MoP single crystals. (a) Schematic of the CVD approach for synthesizing 2D MoP single crystals at ambient pressure. (b) A typical SEM image of the 2D MoP crystals. (c) A typical AFM image of the 2D MoP crystal. The inset shows the corresponding thickness profile corresponding to the region marked by a white line. (d) EDX elemental mapping of $\mathrm{P}$ and Mo of a hexagonal MoP single crystal.

surface, thus to fabricate ultrathin MoP single crystals (Fig. S1). SEM and AFM characterizations were employed to investigate the morphology and thickness of the obtained 2D MoP crystals. SEM image of MoP crystals (Fig. 1b) shows the hexagonal shape. From the EDX mapping (Fig. S2), P and Mo exhibit homogeneous distribution, while $\mathrm{Ga}$ is not detected. In addition, the corresponding EDX spectrum of MoP shows the counts of $\mathrm{P}$ and $\mathrm{Mo}$ are $100 \%$, and the atomic ratio of $\mathrm{P}$ and $\mathrm{Mo}$ is about 1:1 (Table S1). These experimental results show the high purity of 2D MoP. EDX elemental mapping images collected from TEM of Mo and P for MoP crystal are also shown in Fig. 1d. The dark spots in EDS mapping might be caused by radiation damage of e-beam irradiation [21], which results in the damage of samples and appearance of some dark spots [22]. The thickness of MoP was detected by AFM, revealing a thickness of $10 \mathrm{~nm}$ (Fig. 1c). Controlling growth time can obtain $2 \mathrm{D}$ MoP single crystals with different thicknesses (Fig. S3). In addition, XPS was employed to survey the surface electronic state of each element in MoP crystals. The P $2 p$ exhibits two distinct peaks observed at 129.2 and $130.1 \mathrm{eV}$, representing the $P$ $2 \mathrm{p}_{3 / 2}$ and P $2 \mathrm{p}_{1 / 2}$ related to P bonded with Mo (Fig. S4a), respectively [23]. The Mo $3 \mathrm{~d}$ spectrum exhibits the peaks at 231.1 and $227.9 \mathrm{eV}$, which can be designated as Mo in the MoP (Fig. S4b) [24]. XPS result of the fitted Ga $2 \mathrm{p}_{3 / 2}$ spectra is shown in Fig. S4c. The main peak centered at binding energy of $1118.9 \mathrm{eV}$ results from the $\mathrm{Ga}_{2} \mathrm{O}_{3}$ at $\mathrm{Ga}$ surface. The peak located at $1118.2 \mathrm{eV}$ is due to $\mathrm{Ga}_{2} \mathrm{O}$ at
Ga surface. The peak located at $1116.8 \mathrm{eV}$ corresponds to metal state $\mathrm{Ga}^{0}$ [25]. The results are coincident with the fact that $\mathrm{Ga}$ spontaneously forms an oxide layer under ambient conditions [26]. The peak of Ga-P $(1117.3 \mathrm{eV})$ is not detected [27], also indicating the high purity of the prepared MoP. Moreover, Raman spectrum of MoP single crystals was performed with one notable peak at $409 \mathrm{~cm}^{-1}$ (Fig. S5). According to the calculation, the $\mathrm{E}$ mode is Raman active due to the absence of inversion symmetry in MoP crystal [28]. Raman spectrum of MoP single crystals is similar to that in the reported study [29].

Crystal structure and crystalline quality of the prepared MoP samples were evaluated by HRTEM and STEM. Low-magnification HAADF-STEM image of the obtained hexagonal MoP crystal is given in Fig. 2a. Selected area electron diffraction (SAED) collected on this sample in Fig. $2 \mathrm{~b}$ demonstrates only one set of hexagonally arranged diffraction spots, confirming the hexagonal-symmetry atomic arrangement and single-crystalline characteristic of the MoP [30]. The typical atomic model of (001) plane from the hexagonal MoP with $D_{3 h}$ symmetry is displayed in Fig. 2c. Fig. 2d, e display the atomic-level HAADFSTEM image and BF-STEM image of the 2D MoP single crystal, respectively. In Fig. 2d, the brighter spots are the Mo atoms and the dimmer spots are the $\mathrm{P}$ atoms. The hexagonal lattice consisting of $\mathrm{P}$ and Mo atoms can be identified, which agrees well with the $D_{3 h}$ symmetry indicated in Fig. 2c. The nearly perfect atomic structure reveals a lattice spacing of $0.32 \mathrm{~nm}$, in parallel with (001) 

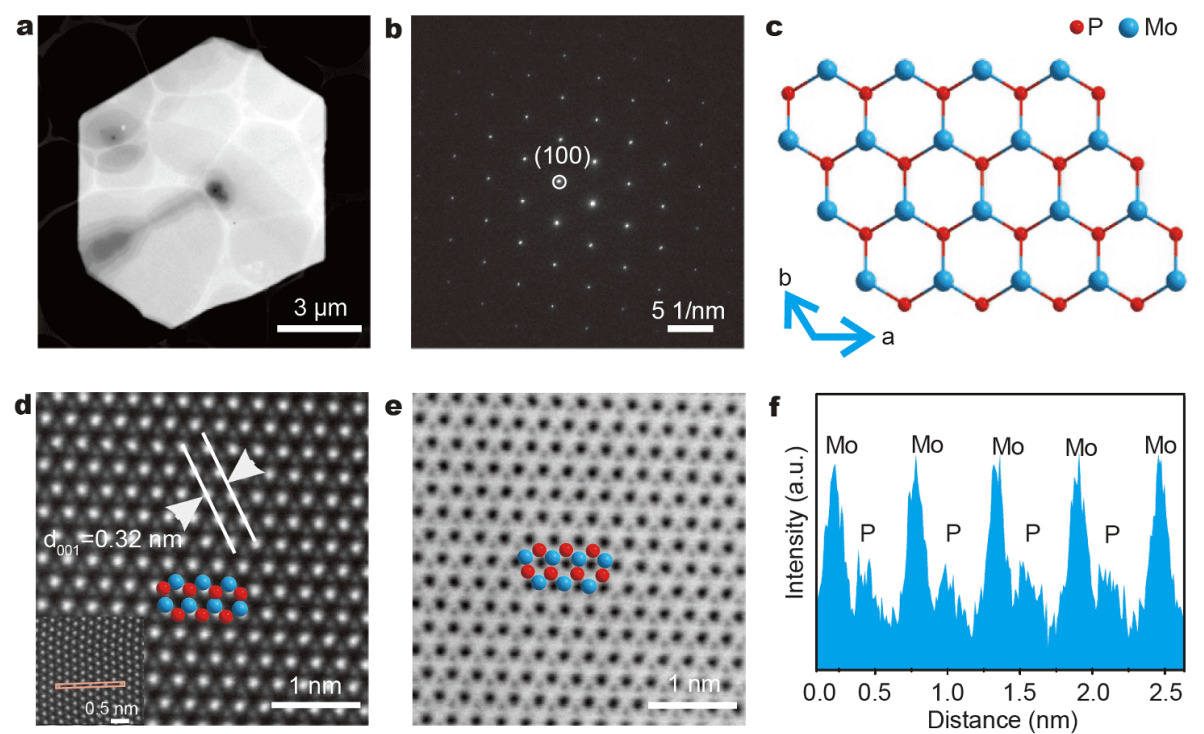

Figure 2 Characterization of atomic structure of 2D MoP single crystal. (a) Low-magnification HAADF-STEM image of a 2D MoP single crystal. (b) SAED pattern collected from the 2D MoP single crystal. (c) Scheme of the (001) face structure of MoP. (d, e) HAADF- and BF-STEM image of the 2D MoP single crystal, respectively. (f) Intensity profile of the selected area marked in (d) by a yellow rectangle.

planes in MoP [9]. Fig. $2 \mathrm{f}$ exhibits the intensity profile corresponding to the region marked in the inset of Fig. $2 \mathrm{~d}$ by a yellow rectangle, which shows the intensity dispersion of Mo and $\mathrm{P}$ atoms. The uniform intensity differences between the bright and dim spots reveal that there are no vacancies or defects [31]. Furthermore, the HAADF intensity ratio of Mo and $\mathrm{P}$ is consistent with the value of $Z_{\mathrm{Mo}}{ }^{1.7} / Z_{\mathrm{P}}{ }^{1.7}$, which agrees well with the previous report [32]. Fig. 2e is the BF-STEM image of the $2 \mathrm{D}$ MoP single crystal and the brightness of the atoms is reversed, which can also clearly show the perfect hexagonal crystal structure of the 2D MoP. These characterizations indicate the as-prepared 2D MoP samples display high crystalline quality.

SHG refers to an NLO effect that two photons with the same frequency encounter with nonlinear materials producing one double frequency photon (Fig. 3a) [33]. The experimental setup of SHG measurement is shown in Fig. S6. We employed it to investigate the second-order NLO property and the potential applications in optoelectronics fields, and also to explore the structural information or crystal configuration of MoP single crystal. The nonlinear spectra of MoP were measured at different excitation wavelengths (Fig. 3b), showing that the SHG signal reaches a maximum value at the incident laser wavelengths of $860 \mathrm{~nm}$. Excitation power dependence of SHG intensity was investigated at the incident laser with the power from 1 to $7.5 \mathrm{~mW}$ under the excitation wave- length of $860 \mathrm{~nm}$. In Fig. 3c, SHG signals can be observed at $430 \mathrm{~nm}$ displaying the second-order nonlinear process. Additionally, laser power dependence of SHG intensity is plotted in natural logarithm, displaying a fitting slope of 2.2 (Fig. 3d), close to the theoretically calculated value of 2 , revealing the distinct quadratic relationship between the SHG intensity and excitation power [34]. Meanwhile, the homogeneous distribution of SHG mapping in Fig. 3e manifests the high quality and homogenous thickness of 2D MoP. We further studied the angle-dependent SHG intensity of MoP single crystal, because the polarizationresolved SHG depends on the structural symmetry. The structure of MoP belongs to the $D_{3 h}$ point group, the function of the angle-dependent SHG intensity can be theoretically predicted as $I=I_{0} \cdot \cos ^{2}(3 \theta)$, where $\theta$ represents the azimuthal angle between the incident laser polarization and the mirror plane of a crystal, $I$ and $I_{0}$ represent the detected SHG signal and the maximum SHG signal, respectively [35]. We observe a typical sixfold pattern with the azimuthal angle from $0^{\circ}$ to $360^{\circ}$ (Fig. 3f), further displaying the threefold symmetry of $\mathrm{MoP}$ crystal, in accordance with the HAADF-STEM image and the $D_{3 h}$ crystal structure.

To quantitatively investigate the SHG properties of MoP, we calculated the SHG susceptibility of MoP by using DFT calculation. Fig. 4a shows the dispersion of the NLO susceptibilities with different photon energies. Because the structure of MoP belongs to the $D_{3 h}$ point 

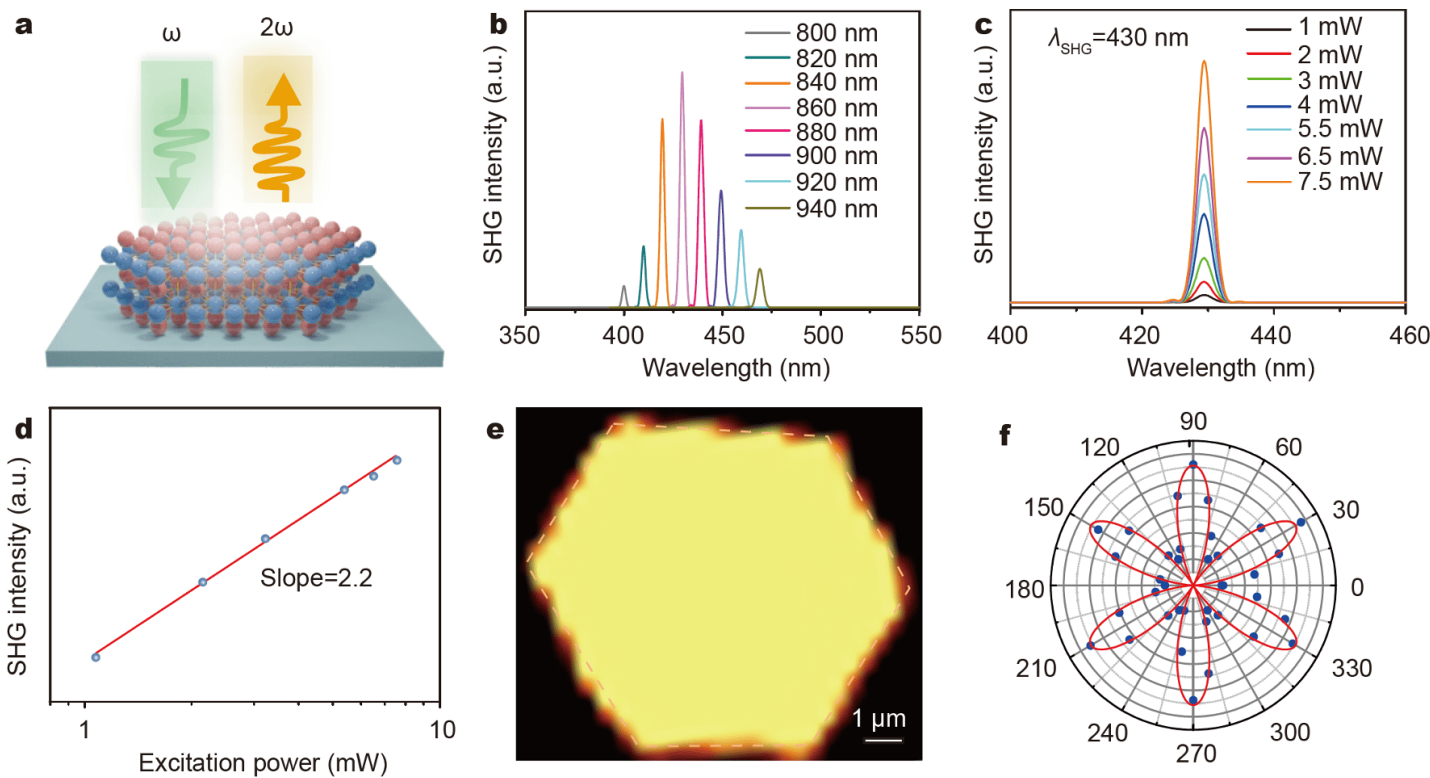

Figure 3 Investigation of SHG of the as-synthesized 2D MoP single crystal. (a) Schematic diagram of an SHG process. (b) Excitation wavelengthdependent SHG spectra. (c) Excitation power-dependent SHG spectra. (d) The corresponding linear fitting in logarithmic coordinates. (e) SHG mapping of a typical hexagonal MoP single crystal. (f) Polarization-angle-dependent SHG intensity of 2D MoP single crystal.
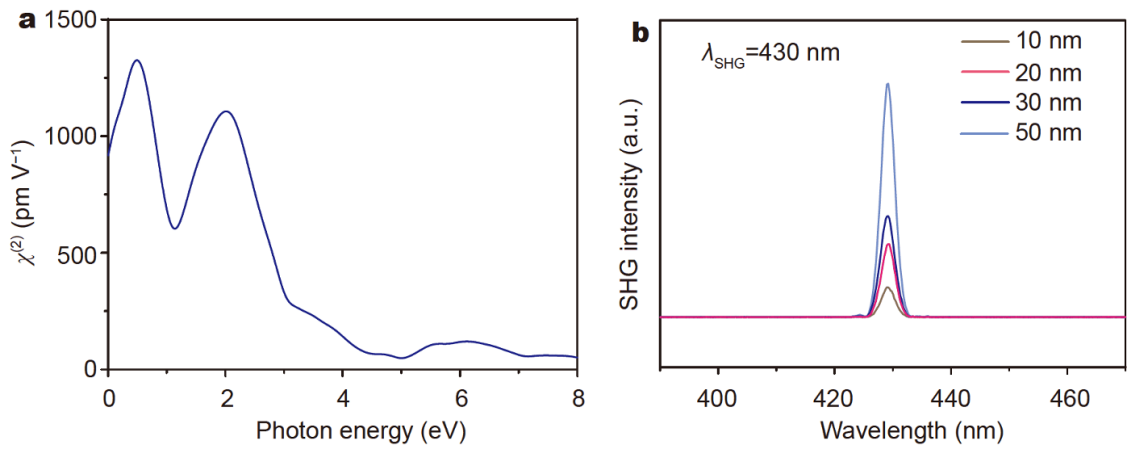

Figure 4 (a) Dispersion of the second-order nonlinear optical susceptibilities of MoP with different photon energies derived from DFT calculation. (b) SHG intensity of MoP single crystals with different thicknesses.

group, there is only one independent component [36]:

$\chi^{(2)}=\chi_{x x t}^{(2)}=\chi_{x y x}^{(2)}=\chi_{y x x}^{(2)}=-\chi_{y y y}^{(2)}$

The calculated SHG susceptibility of MoP at $860 \mathrm{~nm}$ is about $815 \mathrm{pm} \mathrm{V}^{-1}$, which makes it a promising candidate as ultrathin frequency doubling crystal. We also compared the second-order NLO susceptibility of MoP with other reported typical 2D nonlinear optical crystals and commercially available nonlinear optical crystals (Table S2). The comparison results also indicate the high second-order NLO susceptibility of 2D MoP. In addition, the thickness-dependent SHG signals of $2 \mathrm{D} \mathrm{MoP}$ at an excitation wavelength of $860 \mathrm{~nm}$ were investigated, as shown in Fig. 4b. SHG intensity of 2D MoP gets further intensified as the sample thickness increases from 10 to $50 \mathrm{~nm}$, which is consistent with the reported study [37]. Such a characteristic of MoP plays a crucial role in the NLO field since it enables a stable frequency conversion efficiency under different thicknesses [37].

\section{CONCLUSIONS}

In summary, we report the growth of ultrathin 2D MoP single crystals on liquid metals by CVD. Liquid Ga possesses an atomically smooth surface, which is one of the most suitable growth substrates to synthesize $2 \mathrm{D}$ materials. The obtained 2D MoP single crystals with a thickness of $10 \mathrm{~nm}$ display high quality. Next, we investigated the intrinsic strong SHG effect of MoP for the first time, 
displaying that $2 \mathrm{D} \mathrm{MoP}$ is the promising ultrathin frequency doubling crystal. Our study provides an approach to synthesize and explore many other 2D TMPs for future applications.

Received 10 June 2020; accepted 18 September 2020; published online 13 November 2020

1 Novoselov KS, Geim AK, Morozov SV, et al. Electric field effect in atomically thin carbon films. Science, 2004, 306: 666-669

2 Tan L, Wang C, Zeng M, et al. Graphene: An outstanding multifunctional coating for conventional materials. Small, 2017, 13: 1603337

3 Mendes RG, Ta HQ, Yang X, et al. In situ $\mathrm{N}$-doped graphene and Mo nanoribbon formation from $\mathrm{Mo}_{2} \mathrm{Ti}_{2} \mathrm{C}_{3}$ MXene monolayers. Small, 2020, 16: 1907115

4 Si J, Zeng M, Ta HQ, et al. Adsorption-free growth of ultra-thin molybdenum membranes with a low-symmetry rectangular lattice structure. Small, 2020, 16: 2001325

5 Shao Y, Shi X, Pan H. Electronic, magnetic, and catalytic properties of thermodynamically stable two-dimensional transition-metal phosphides. Chem Mater, 2017, 29: 8892-8900

6 Li R, Duan Y. Structural and anisotropic elastic properties of hexagonal MP ( $\mathrm{M}=\mathrm{Ti}, \mathrm{Zr}, \mathrm{Hf})$ monophosphides determined by first-principles calculations. Philos Mag, 2016, 96: 3654-3670

7 Lv BQ, Feng ZL, Xu QN, et al. Observation of three-component fermions in the topological semimetal molybdenum phosphide. Nature, 2017, 546: 627-631

8 Kumar N, Sun Y, Nicklas M, et al. Extremely high conductivity observed in the triple point topological metal MoP. Nat Commun, 2019, 10: 2475

9 Liang K, Pakhira S, Yang Z, et al. S-doped MoP nanoporous layer toward high-efficiency hydrogen evolution in $\mathrm{pH}$-universal electrolyte. ACS Catal, 2018, 9: 651-659

10 Chi Z, Chen X, An C, et al. Pressure-induced superconductivity in MoP. npj Quant Mater, 2018, 3: 28

11 Guo SD. Anisotropic lattice thermal conductivity in three-fold degeneracy topological semimetal MoP: A first-principles study. J Phys-Condens Matter, 2017, 29: 435704

12 Jiang $\mathrm{Y}, \mathrm{Lu} \mathrm{Y}$, Lin J, et al. A hierarchical MoP nanoflake array supported on Ni foam: A bifunctional electrocatalyst for overall water splitting. Small Methods, 2018, 2: 1700369

13 Xing Z, Liu Q, Asiri AM, et al. Closely interconnected network of molybdenum phosphide nanoparticles: A highly efficient electrocatalyst for generating hydrogen from water. Adv Mater, 2014, 26: 5702-5707

14 Shi Y, Li H, Li LJ. Recent advances in controlled synthesis of twodimensional transition metal dichalcogenides via vapour deposition techniques. Chem Soc Rev, 2015, 44: 2744-2756

15 Kalantar-Zadeh K, Tang J, Daeneke T, et al. Emergence of liquid metals in nanotechnology. ACS Nano, 2019, 13: 7388-7395

16 Chen Y, Liu K, Liu J, et al. Growth of 2D GaN single crystals on liquid metals. J Am Chem Soc, 2018, 140: 16392-16395

17 Wang J, Chen L, Wu N, et al. Uniform graphene on liquid metal by chemical vapour deposition at reduced temperature. Carbon, 2016, 96: 799-804

18 Zeng M, Fu L. Controllable fabrication of graphene and related two-dimensional materials on liquid metals via chemical vapor deposition. Acc Chem Res, 2018, 51: 2839-2847
19 Sun X, Zhao S, Bachmatiuk A, et al. 2D intrinsic ferromagnetic MnP single crystals. Small, 2020, 16: 2001484

20 Jiang X, Zhang L, Liu S, et al. Ultrathin metal-organic framework: An emerging broadband nonlinear optical material for ultrafast photonics. Adv Opt Mater, 2018, 6: 1800561

21 Egerton RF, Li P, Malac M. Radiation damage in the TEM and SEM. Micron, 2004, 35: 399-409

22 Hobbs LW. Electron-beam sensitivity in inorganic specimens. Ultramicroscopy, 1987, 23: 339-344

23 Xiao P, Sk MA, Thia L, et al. Molybdenum phosphide as an efficient electrocatalyst for the hydrogen evolution reaction. Energy Environ Sci, 2014, 7: 2624-2629

24 Sun X, Lu L, Zhu Q, et al. MoP nanoparticles supported on indium-doped porous carbon: outstanding catalysts for highly efficient $\mathrm{CO}_{2}$ electroreduction. Angew Chem Int Ed, 2018, 57: 24272431

25 Scharmann F, Cherkashinin G, Breternitz V, et al. Viscosity effect on GaInSn studied by XPS. Surf Interface Anal, 2004, 36: 981-985

26 Zavabeti A, Ou JZ, Carey BJ, et al. A liquid metal reaction environment for the room-temperature synthesis of atomically thin metal oxides. Science, 2017, 358: 332-335

27 Wilkins SJ, Paskova T, Reynolds Jr. CL, et al. Comparison of the stability of functionalized GaN and GaP. ChemPhysChem, 2015, 16: $1687-1694$

28 Chen J. Unconventional superconductivity in the topological semimetal MoP: Evidence from first-principles calculated electron-phonon coupling. Comput Mater Sci, 2020, 173: 109466

29 Ren JT, Chen L, Weng CC, et al. Ultrafine molybdenum phosphide nanocrystals on a highly porous N,P-codoped carbon matrix as an efficient catalyst for the hydrogen evolution reaction. Mater Chem Front, 2018, 2: 1987-1996

30 Shi J, Wang X, Zhang S, et al. Two-dimensional metallic tantalum disulfide as a hydrogen evolution catalyst. Nat Commun, 2017, 8: 958

31 Cui F, Zhao X, Xu J, et al. Controlled growth and thickness-dependent conduction-type transition of $2 \mathrm{D}$ ferrimagnetic $\mathrm{Cr}_{2} \mathrm{~S}_{3}$ semiconductors. Adv Mater, 2020, 32: 1905896

32 Chiu KC, Huang KH, Chen CA, et al. Synthesis of in-plane artificial lattices of monolayer multijunctions. Adv Mater, 2018, 30: 1704796

33 Gong C, Chu J, Yin C, et al. Self-confined growth of ultrathin 2D nonlayered wide-bandgap semiconductor $\mathrm{CuBr}$ flakes. Adv Mater, 2019, 31: 1903580

34 Wang $\mathrm{R}$, Liang F, Wang F, et al. $\mathrm{Sr}_{6} \mathrm{Cd}_{2} \mathrm{Sb}_{6} \mathrm{O}_{7} \mathrm{~S}_{10}$ : Strong SHG response activated by highly polarizable $\mathrm{Sb} / \mathrm{O} / \mathrm{S}$ groups. Angew Chem Int Ed, 2019, 58: 8078-8081

35 Hu X, Huang P, Jin B, et al. Halide-induced self-limited growth of ultrathin nonlayered Ge flakes for high-performance phototransistors. J Am Chem Soc, 2018, 140: 12909-12914

36 Yin X, Ye Z, Chenet DA, et al. Edge nonlinear optics on a $\mathrm{MoS}_{2}$ atomic monolayer. Science, 2014, 344: 488-490

37 Zhou N, Gan L, Yang R, et al. Nonlayered two-dimensional defective semiconductor $\gamma-\mathrm{Ga}_{2} \mathrm{~S}_{3}$ toward broadband photodetection. ACS Nano, 2019, 13: 6297-6307

Acknowledgements The research was supported by the National Natural Science Foundation of China (21673161 and 21905210) and the Sino-German Center for Research Promotion (GZ 1400).

Author contributions $\mathrm{Fu} \mathrm{L}$ and Zeng $\mathrm{M}$ developed the concept and 
conceived the experiments. Cao F, Zheng S and Ding Y performed the main experiments. Liang J conducted the AFM characterization. Li Z conducted the DFT calculations. Wang $\mathrm{Z}$ and Wei $\mathrm{B}$ conducted the TEM characterization. Cao F wrote the manuscript. Fu L, Xu N and Zeng $\mathrm{M}$ revised the manuscript. All of the authors contributed to the data analysis and scientific discussion.

Conflict of interest The authors declare no conflict of interest.

Supplementary information Supporting data are available in the online version of the paper.

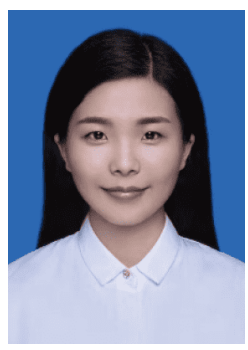

Feifei Cao received her Master degree from Chongqing University in 2018. She is now a PhD candidate under the supervision of Prof. Lei Fu and Prof. Nan Xu at Wuhan University. Her current research interest is the controllable growth of 2D materials.

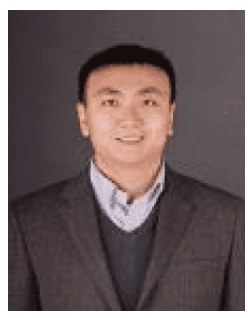

Nan Xu received his BSc degree from Harbin Institute of Technology in 2007. He obtained his $\mathrm{PhD}$ degree from the Institute of Physics, Chinese Academy of Sciences in 2013. Then he worked as a Postdoctoral Fellow at Paul Scherrer Institute, Swiss Light Source, Switzerland (20132017). In 2017, he joined Wuhan University as a full professor. His current interests of research relate to the condensed matter physics.

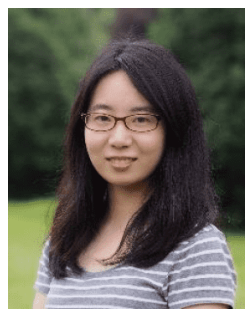

Mengqi Zeng received her BSc and PhD degrees from Wuhan University in 2013 and 2018, respectively. Then, she joined Wuhan University as an associate professor. Her current research interests are the catalyst design for controllable growth and self-assembly of $2 \mathrm{D}$ materials.

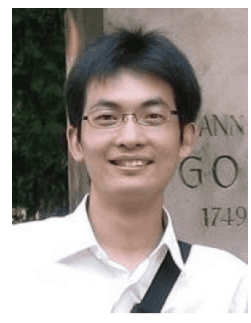

Lei $\mathrm{Fu}$ received his $\mathrm{PhD}$ degree from the Institute of Chemistry, Chinese Academy of Sciences in 2006. After that, he worked as a Director's Postdoctoral Fellow at Los Alamos National Laboratory, Los Alamos, New Mexico (2006-2007). Thereafter, he became an Associate Professor at Peking University. In 2012, he joined Wuhan University as a full professor. His current interests of research relate to controlled growth and novel property exploration of $2 \mathrm{D}$ atomic layer thin crystals.

\section{化学气相沉积法在液态金属上制备2D MoP单晶}

曹菲菲 ${ }^{1}$, 郑舒婷 ${ }^{2}$, 梁晶晶 ${ }^{1}$, 李志 ${ }^{3}$, 卫斌, 丁一然, 王中长 ${ }^{4}$, 曾梦琪 ${ }^{2^{*}}$, 徐楠 ${ }^{*}$, 付磊 ${ }^{1,2}$

摘要 二维过渡金属磷化物 (TMPs) 有许多新奇的性质和应用. 作 为二维TMPs的一员, 二维 MoP有许多独特的物理化学性质. 然而 由于缺乏制备二维MoP的方法, 目前还未成功制备二维 MoP, 因此 限制了对二维 $\mathrm{MoP}$ 众多性质的探索. 本文采用化学气相沉积法在 液态金属镓 $(\mathrm{Ga})$ 上制备了厚度为 $10 \mathrm{~nm}$ 的二维 $\mathrm{MoP}$ 单晶. 液态 $\mathrm{Ga}$ 具 有原子级平整的表面, 能作为制备二维材料的合适生长基底. 在生 长过程中, Mo源扩散到 $\mathrm{Ga}$ 表面与磷源反应, 从而在 $\mathrm{Ga}$ 表面反应得 到二维 $\mathrm{MoP}$ 单晶. 此外, 由于二维 $\mathrm{MoP}$ 具有本征的非中心对称结构, 文中首次研究了二维 $\mathrm{MoP}$ 的二次谐波信号的产生. 本文为其他二 维TMPs的制备和性质探索提供了新思路. 